\title{
Hunter syndrome revisited
}

\author{
Dan Cristian Gheorghe ${ }^{1,5}$, Adina Zamfir-Chiru-Anton ${ }^{2}$, Elisabeta Loredana Ene ${ }^{3}$, \\ Adina Elena Stanciu 4,5 \\ ${ }^{1}$ ENT Department, "MS Curie" Hospital, Bucharest, Romania \\ 2ENT Department, "Gr. Alexandrescu“ Hospital, Bucharest, Romania \\ ${ }^{3}$ ENT Department, City Hospital, Slatina, Romania \\ ${ }^{4}$ Department of Carcinogenesis and Molecular Biology, Institute of Oncology, Bucharest, Romania \\ 5"Carol Davila" University of Medicine and Pharmacy, Bucharest, Romania
}

\begin{abstract}
Hunter syndrome is the type II of mucopolysaccharidose. With impaired glycosaminoglycan catabolism, heparan and dermatan sulfate accumulate in lysosomes. Multiple organ dysfunction is a consequence of this effect. We present the case of a a 9 year-old boy was refered to our department for breathing difficulties, discussing the diagnosis and treatment challenges of the case.
\end{abstract}

Keywords: Hunter syndrome, mucopolysaccharidose, glycosaminoglycan

\section{INTRODUCTION}

Hunter syndrome is the type II of mucopolysaccharidose. It is a X-linked genetic disease. The main defect is the mutation of the IDS gene, with resultant iduronate-2-sulfatase (I2S) deficiency. With impaired glycosaminoglycan catabolism, heparan and dermatan sulfate accumulate in lysosomes. Multiple organ dysfunction is a consequence of this effect.

Incidence of disease is cited at 1.3 in 100,000 live births [1]. It occurs almost exclusively in males.

Disease manifest clinically with neurological disorders, skeletal deformities, cardiomyopathy and airway obstruction. Developmental delay and intellectual disability can be found in two-thirds of patients [2]. Shortened life expectancy is due to cardiac and respiratory involvement and life expectancy is around 15 years [3].

Management includes enzyme replacement (ERT) and symptomatic support of different systemic manifestations. Auto-antibodies can be developed by the patients in response to ERT. Stem cell transplantation is in research phase as well. Early diagnosis can prove beneficial [4]. Newborn screening is already in practice in US, together with other lysosomal diseases. Long term benefit of these programs remain to be demonstrated [5].

We present a case that was admitted in our department and therapeutical challenges that we needed to approach.

\section{CASE PRESENTATION}

A 9 year-old boy was referred to our department for breathing difficulties.

From history we note high body mass index and a particular face appearance.

Adenoid and tonsil surgery removement had been performed a few month before current referral. Sleep disordered breathing persisted inspite of surgery, so we performed several other investigations. Results of the lab test (detecting urinary glycosaminoglycans level and measuring I2S activity in plasma or dry blood spots). Other investigations that helped in case aproach were spirometry (forced vital capacity - FVC) and walking motor performance (6 min.). Both were compatible with clinical manifestation of Hunter syndrome. 
He had been diagnosed with mucopolysaccharidose type II (Hunter syndrome) and had a long term tracheostomy.

We performed CT imaging to reveal possible causes of acute airway obstruction. Pulmonary fields showed no abnormalities. A diffuse diminishing of the pharyngeal and tracheal airways could be elicited (figures 1a-e).

Patient was investigated by tracheal endoscopy under general anesthesia (GA). The images showed variate thickening of the tracheal wall giving the appearance of granulation tissue and obstruction of the airway (figures $2 \mathrm{a}-\mathrm{c}$ ).

Due to the obstructive nature of the tracheal wall, we decided that best management approach would be a long tracheal cannula use, so that the respiratory support bypasses the stenotic segments of the airway. Medication as antibiotic prophylaxis and prompt infection assessment would ensure a proper diameter of the distal trachea and bronchi.

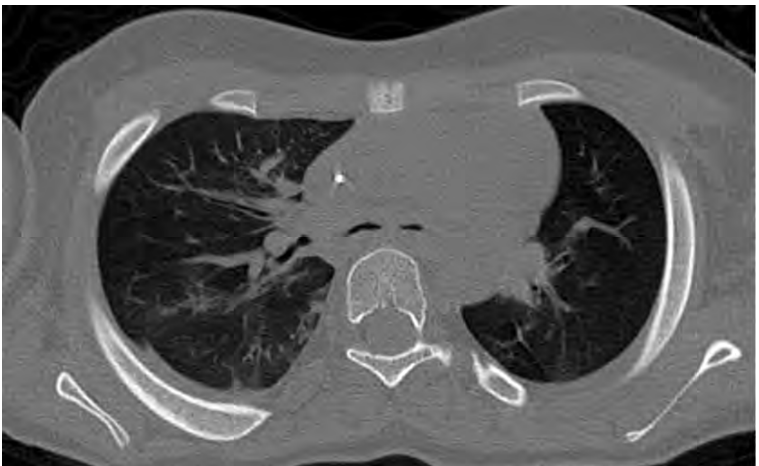

$1 a$

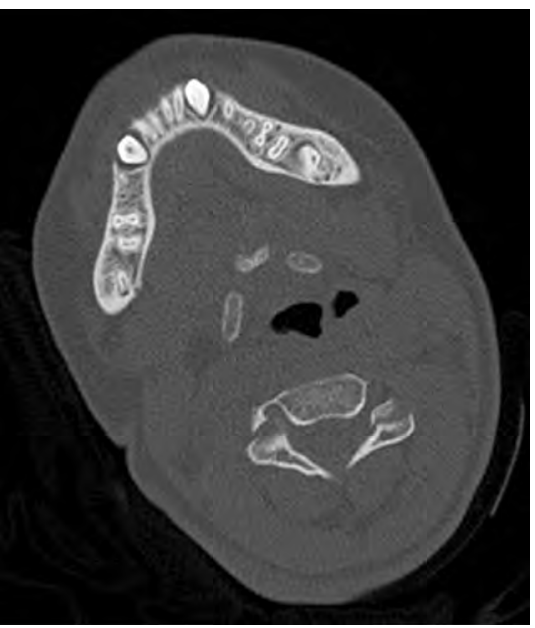

1c (hypopharynx)

$1 d$

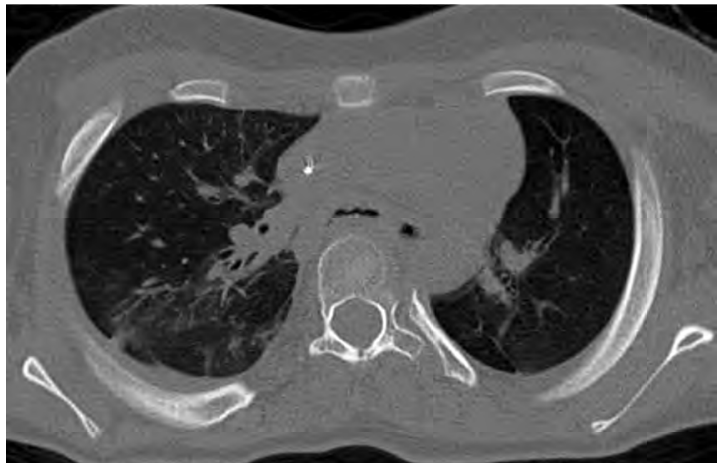

$1 b$

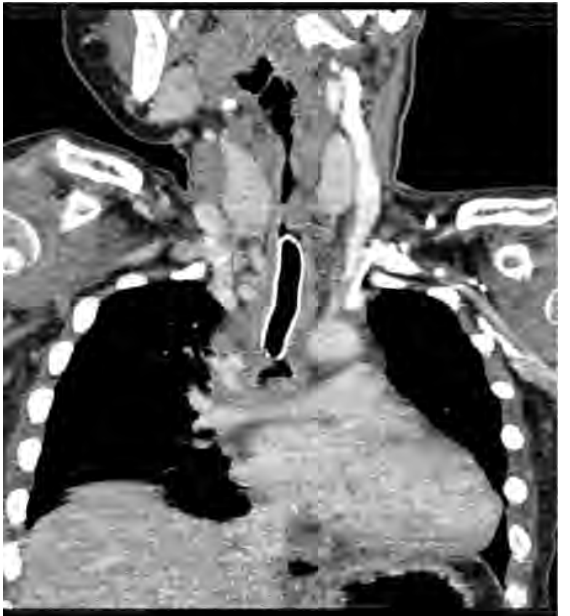

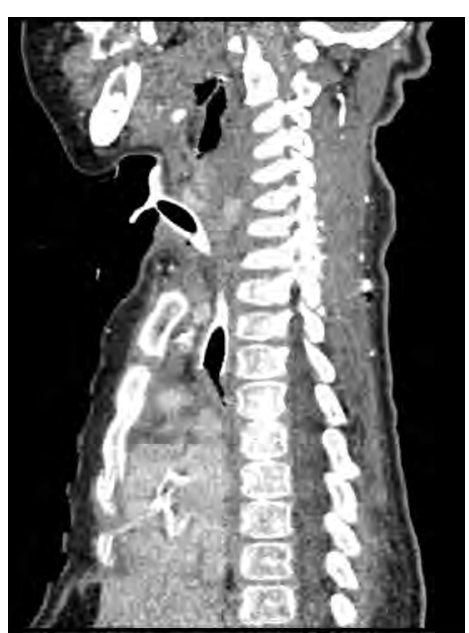

$1 e$

FIGURES 1a-e. Tracheal and pharyngeal airways

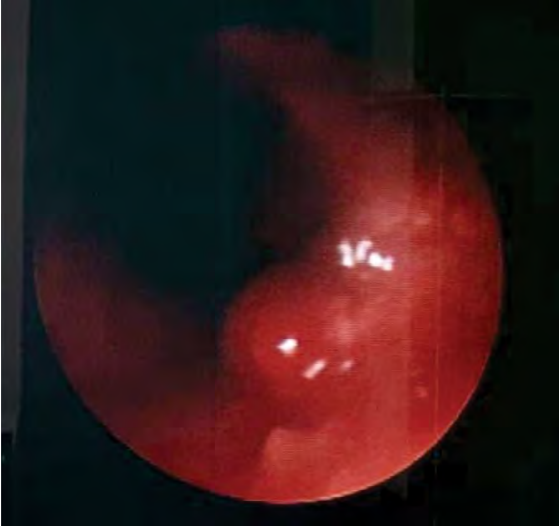
FIGURES 2a,b,c. Tracheal endoscopy

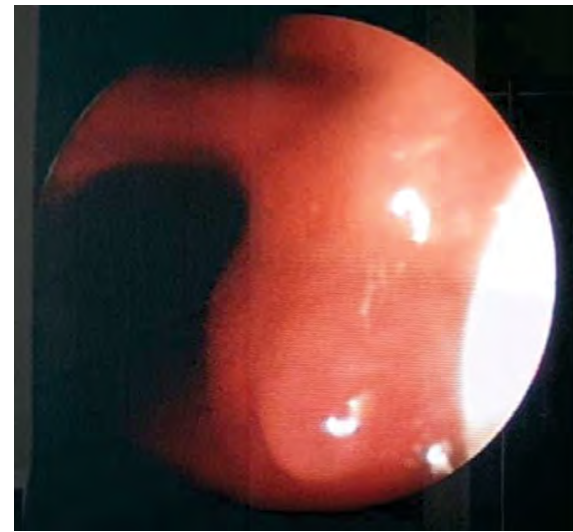

$b$

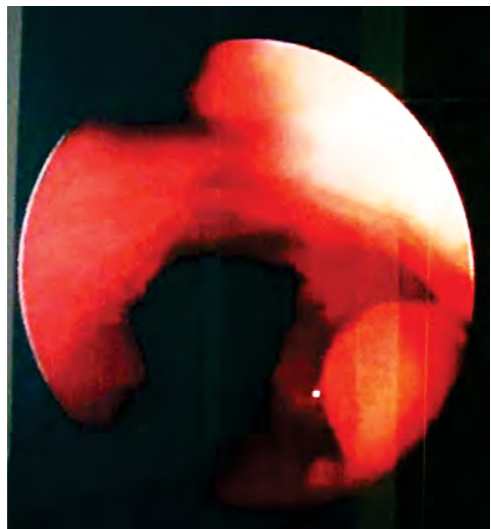

C 


\section{DISCUSSION}

Hunter syndrome is a genetic disease with over 350 mutations of the IDS gene described to date. Some therapeutic options are available but early diagnosis is still compulsory, in order to monitor the patient and prevent long lasting mucoplysaccharid acumulation.

Clinical features of the disease do not manifest until 2 to 4 years of age. Full lips, broad nose and macroglossia still account for most of the prominent signs. Other symptoms can be cited: hoarse voice and sleep disordered breathing, hydrocephalus, hepatosplenomegaly and abdominal wall hernia. Carpal tunnel syndrome, spinal stenosis and heart valve problems can be seen in these patients. Multidisciplinary approach is advisable [6].

Skeletal abnormalities include joint deformities (stiffness and contractures) and thickening of multiple long bones (dysostosis multiplex). Short stature is associated.

Intellectual disability can be found in severe forms of the disease and start to manifest after 6 years of age.

Affected individuals need ENT monitoring for recurrent or chronic otitis media and hearing loss. Airway problems are common in MPS II. Some authors noted that $32 \%$ of infants with Hunter syndrome present neonatal airway distress [7]. Endoscopy typically shows redundant oropharyngeal mucosa, laryngomalacia, tracheomalacia. Airway obstruction is typical. Management of these features can be difficult and eventually progress in spite of proper treatment $[5,8]$.

Diagnosis is established by screening urinary glycosaminoglycans (GAGs) and measuring I2S activity in plasma or dry blood spots. Monitoring

\section{REFERENCES}

1. Baehner $F$ et al. Cumulative incidence rates of the mucopolysaccharidoses in Germany. J Inherit Metab Dis. 2005. 28(6):1011-7.

2. Holt JB, Poe MD, Escolar ML. Natural progression of neurological disease in mucopolysaccharidosis type II. Pediatrics, 2011. 127(5):e1258-65.

3. Jones $S$ et al. Profile of natural history in 104 patients with mucopolysaccharidosis type II: Insights from the Hunter Outcome Survey (HOS). Molecular Genetics and Metabolism, 2018. 123(2):S73.

4. Lampe $C$ et al. Enzyme Replacement Therapy in Mucopolysaccharidosis II Patients Under 1 Year of Age. JIMD Rep, 2014. 14:99-113.

5. Broomfield, A et al. Ten years of enzyme replacement therapy in paediatric onset mucopolysaccharidosis II in England. Mol Genet Metab, 2020. 129(2):98-105.

6. Scarpa, M et al. Mucopolysaccharidosis type II: European recommendations for the diagnosis and multidisciplinary management of a rare disease. Orphanet J Rare Dis, 2011. 6:72.

7. Dodsworth C, Burton BK. Increased incidence of neonatal respiratory distress in infants with mucopolysaccharidosis type II (MPS II, Hunter syndrome). Mol Genet Metab. 2014. 111(2):203-4. the disease could also be done by using heparan sulphate and EGF blood levels [9]. Other investigations that can be useful are spirometry (forced vital capacity - FVC) and walking motor performance (6 min.).

Enzyme replacement therapy (ERT) relies on iduronate sulphatase administration, but results still need a long term assessment. Earlier introduction of the enzyme is safe and better results are expected [4]. A decrease in spleen and liver volume were noted after this therapeutic apprach. Motor performance of the patient seems to improve, but central nervous system does not. The risc of death is lowered after enzyme replacement therapy. Respiratory disfunction, cardiac and skeletal problems are not improving after ERT [5].

Stem cell transplantation is assessed at the moment, with somewhat better results than ERT $[9,10]$.

Symptoms management in Hunter syndrome can be difficult and prone to complications. Medical visits and hospital admissions are commonly seen in children with the disease [11]. Surgery is frequent in early years of age [3]. Problems should be addressed in facilities used to work with difficult airway. Death in MPS II patients seem to be caused mainly by respiratory failure [3]. Cases with severe neurological impairment have a worse prognosis [12].

\section{CONCLUSIONS}

Teamwork is essential in therapeutic approach of MPS II patients. Genetic studies should be available and a high degree of suspicion could better detect symptoms of disease. The different management protocols should be detailed to the parents of the afflicted children. Possible outcomes/limitations can also be presented early in the course of the disease.

8. Bordley $\mathrm{M}$ et al. Long-term clinical outcomes of patients with mucopolysaccharidosis type II: A case series. Molecular Genetics and Metabolism, 2020. 129(2):S32.

9. Fujitsuka $\mathrm{H}$ et al. Biomarkers in patients with mucopolysaccharidosis type II and IV. Mol Genet Metab Rep. 2019. 19:100455.

10. Selvanathan A et al. Effectiveness of Early Hematopoietic Stem Cell Transplantation in Preventing Neurocognitive Decline in Mucopolysaccharidosis Type II: A Case Series. JIMD Rep. 2018. 41:81-89.

11. Conner T et al. An online survey of burden of illness in families with mucopolysaccharidosis type II children in the United States. Molecular Genetics and Metabolism Reports, 2019. 21:100499.

12. Jones SA et al. Mortality and cause of death in mucopolysaccharidosis type II-a historical review based on data from the Hunter Outcome Survey (HOS). J Inherit Metab Dis. 2009. 32(4):534-43. 